\title{
Wearable Activity Tracking Device Use in an Adolescent Weight Management Clinic: A Randomized Controlled Pilot Trial
}

\author{
Kanika Bowen-Jallow $\left(\mathbb{D},{ }^{1,2}\right.$ Omar Nunez-Lopez $\mathbb{D}^{1},{ }^{1}$ Alex Wright $\mathbb{D},{ }^{3}$ Erika Fuchs $\mathbb{D}^{\mathbb{D}},{ }^{4}$ \\ Mollie Ahn, ${ }^{3}$ Elizabeth Lyons $\mathbb{D}^{5}{ }^{5}$ Daniel Jupiter $\left(\mathbb{D},{ }^{6}\right.$ Lindsey Berry, ${ }^{1}$ Oscar Suman, ${ }^{1}$ \\ Ravi S. Radhakrishnan $\mathbb{D}^{1,2}$ Andrea M. Glaser, ${ }^{2}$ and Deborah I. Thompson ${ }^{7}$ \\ ${ }^{1}$ Department of Surgery, University of Texas Medical Branch, Galveston, TX, USA \\ ${ }^{2}$ Department of Pediatrics, University of Texas Medical Branch, Galveston, TX, USA \\ ${ }^{3}$ School of Medicine, University of Texas Medical Branch, Galveston, TX, USA \\ ${ }^{4}$ Department of Obstetrics and Gynecology, University of Texas Medical Branch, Galveston, TX, USA \\ ${ }^{5}$ Department of Nutrition and Metabolism, University of Texas Medical Branch, Galveston, TX, USA \\ ${ }^{6}$ Department of Preventive Medicine and Community Health, University of Texas Medical Branch, Galveston, TX, USA \\ ${ }^{7}$ USDA/ARS Children's Nutrition Research Center, Department of Pediatrics, Baylor College of Medicine, Houston, TX, USA
}

Correspondence should be addressed to Kanika Bowen-Jallow; kabowen@utmb.edu

Received 23 April 2020; Revised 2 December 2020; Accepted 30 December 2020; Published 8 January 2021

Academic Editor: Chris I. Ardern

Copyright (C) 2021 Kanika Bowen-Jallow et al. This is an open access article distributed under the Creative Commons Attribution License, which permits unrestricted use, distribution, and reproduction in any medium, provided the original work is properly cited.

\begin{abstract}
Background. The use of physical activity tracker devices has increased within the general population. However, there is limited medical literature studying the efficacy of such devices in adolescents with obesity. In this study, we explored the feasibility of using wearable activity tracking devices as an adjunct intervention on adolescents with obesity. Methods. Randomized controlled pilot trial evaluated the feasibility (attrition $\leq 50 \%$ ) of an activity tracking intervention (ATI) and its effects on weight loss in adolescents with obesity enrolled in an adolescent weight management clinic (AWMC). Outcomes included feasibility (attrition rate) and absolute change in BMI. Differences between groups at 6,12, and 18 weeks were examined. Results. Forty-eight participants were enrolled in the study. Eighteen subjects were randomly assigned to the ATI group and 30 to control. The average age was 14.5 years. Overall, the majority of participants were Hispanic (56\%). Sexes were equally distributed. The average baseline BMI was $37.5 \mathrm{~kg} / \mathrm{m}^{2}$. At the study conclusion, the overall attrition rate was $52.1 \%, 44.4 \%$ in the ATI group versus $56.6 \%$ in the control group, with a differential attrition of $12.2 \%$. The ATI and control groups each showed an absolute decrease in BMI of -0.25 and -2.77 , respectively, with no significant differences between the groups. Conclusion. The attrition rate in our study was $>50 \%$. Participation in the AWMC by the ATI and control groups resulted in maintenance of BMI and body weight for the study duration. However, the use of an activity tracking device was not associated with greater weight loss. This trial is registered with NCT03004378.
\end{abstract}

\section{Introduction}

Over the past few decades, adolescent obesity has developed into an overwhelming public health issue in the United States with $21 \%$ of adolescents suffering from obesity [1-4]. Among Hispanics, Blacks, and Whites, the obesity prevalence in adolescents is $26 \%, 22 \%$, and $14 \%$, respectively [3]. Studies suggest that various demographic factors can lead to variability in weight loss [5-7]. Despite increased clinical efforts and national policies, the weight of the average child in the United States increased by 5 kilograms $(\mathrm{kg})$ in the past three decades [8].

As children with obesity transition into adulthood, the predicted cost of treating subsequent obesity-related comorbidities is significant. An estimated decrease by $1 \%$ of adolescents who suffer from overweight/obesity could save 
between $\$ 463$ and $\$ 691$ million in long-term healthcare costs $[9,10]$. One growing resource in efforts to curb pediatric obesity is multidisciplinary weight management clinics with oversight from physicians, dietitians, psychologists, and physical trainers $[11,12]$.

While patient treatment adherence can be improved through frequent follow-up monitoring provided by multidisciplinary weight loss clinics, patient retention has consistently been identified as a barrier to effective weight management with reported attrition rates varying significantly $(27-73 \%)[13,14]$. Adolescent studies have attempted to identify targeted behavioral interventions to decrease attrition by keeping adolescents and families engaged, which has been met with variable success $[15,16]$. We consider attrition in weight management programs is a relevant indicator of participants' engagement. Activity tracking, as a behavioral technology intervention, may be an avenue to increase (and monitor) engagement. The use of activity tracking devices may allow for decreased attrition rates in adolescent weight management programs.

Wearable fitness trackers have become a prominent component of the effort in initiating behavioral lifestyle modifications to combat obesity $[17,18]$. Activity tracking technology has become increasingly prevalent in the United States, with the wearable fitness industry showing exponential growth within the past few years [19]. Wearable devices have demonstrated accuracy with respect to measurements such as heart rate, step count, move distance, and sleep duration; however, accuracy does deteriorate under conditions of increased activity $[20,21]$. Despite any inaccuracies devices may have, users often report high satisfaction and perception of increased physical fitness [17, 22]. The ease of use, increased utilization, and mobility of activity tracking devices has brought about new opportunities for data collection and subsequent research $[19,23]$.

Studies have shown that activity trackers may promote increased physical activity (PA) and subsequent weight reduction [18, 24-26]. Fitness devices used in conjunction with other health motivation programs can lead to improved weight loss [26, 27], and other studies have studied the benefits and reviewed the effectiveness of utilizing activity tracking in adolescents $[17,28,29]$. A pilot study measuring the effect of activity trackers on physical activity levels in adolescents with obesity demonstrated that parent-adolescent dyads have high correlation in increased physical activity. This finding supports family-centered approaches in weight management interventions [28]. Activity tracking devices have been found to have high satisfaction among adolescents, but also up to $68 \%$ discontinuation rates in short-term follow-up [17]. However, the long-term efficacy of these behavioral changes on better physical health is not fully understood [30]. Furthermore, although PA trackers have increased in prevalence, there is limited medical literature dedicated to assessing the efficacy and feasibility of such devices in adolescents [29].

To our knowledge, there are no randomized trials in the United States assessing the efficacy of activity tracking devices in adolescents enrolled in an adolescent weight management clinic. Because of obesity's resistant nature to many nonsurgical methods, studies on noninvasive interventions that prove successful in sustaining long-term weight loss are highly sought after $[5,31]$. We hypothesized that the adjunct use of activity tracking technology would be feasible among adolescents participating in a multidisciplinary weight management clinic and would result in an absolute decrease in BMI.

\section{Methods}

2.1. Eligibility Criteria. To participate in the study, an adolescent had to (1) be aged between 12 and 18 years, (2) have a BMI greater than or equal to the 95th percentile, with respect to gender-specific BMI-forage growth charts as indicated by the Centers for Disease Control and Prevention (CDC), and (3) be able to speak and read English (Supplement 1). Before enrollment, all the participants self-reported being capable and comfortable using smartphones, personal computers, and/or wearable technology devices. Eligible adolescents also had to be free of acute or lifethreatening diseases and be able to participate in moderate PA. Study enrollment occurred for 12 months. The study was approved by the University of Texas Medical Branch (UTMB) institutional review board (\#16-0241).

\subsection{Adolescent Weight Management Clinic (AWMC).} Adolescents enrolled in the AWMC are 12-18 years of age and have a BMI at or above the 95th percentile. The AWMC is a multidisciplinary clinic where patients and their caretakers meet with a dietitian, pediatric gastroenterologist, pediatric surgeon, and personal fitness instructor every 6-8 weeks. The initial visit includes a thorough assessment, goal setting, dietary and fitness plan design, education, and counseling. Subsequent visits serve to trend progress, address questions and concerns, review and modify diet and exercise plans, reinforce goals, and continued education and counseling. In addition, all participants are instructed to selfmonitor weight at home and are given a scale.

Anthropometric and clinical data are collected at each visit. In addition to dietary and PA goal-setting and tailored guidance, AWMC patients are evaluated for obesity-related illnesses (ORIs), with focus on early identification of nonalcoholic fatty liver disease (NAFLD). Obesity-related biomarkers (ORBs) are measured at baseline and repeated as considered clinically necessary. Patients also undergo liver ultrasound evaluation to screen for hepatomegaly.

2.3. Randomization. Patients in the AWMC at the University of Texas Medical Branch (UTMB) were eligible to enroll in the pilot study if inclusion criteria were met: ages $12-18$ years, $\mathrm{BMI} \geq 95$ th percentile for age and sex group, participant able to read and understand English, and willingness to be randomized to any condition (Supplement 1). Patients were excluded if parental consent or child assent was not obtainable, if the patient could not due to preexisting conditions (e.g., paralysis, heart failure, severe autism or mental retardation, and psychosis), pregnancy, clinical judgment concerning safety, or inability of the participant to 


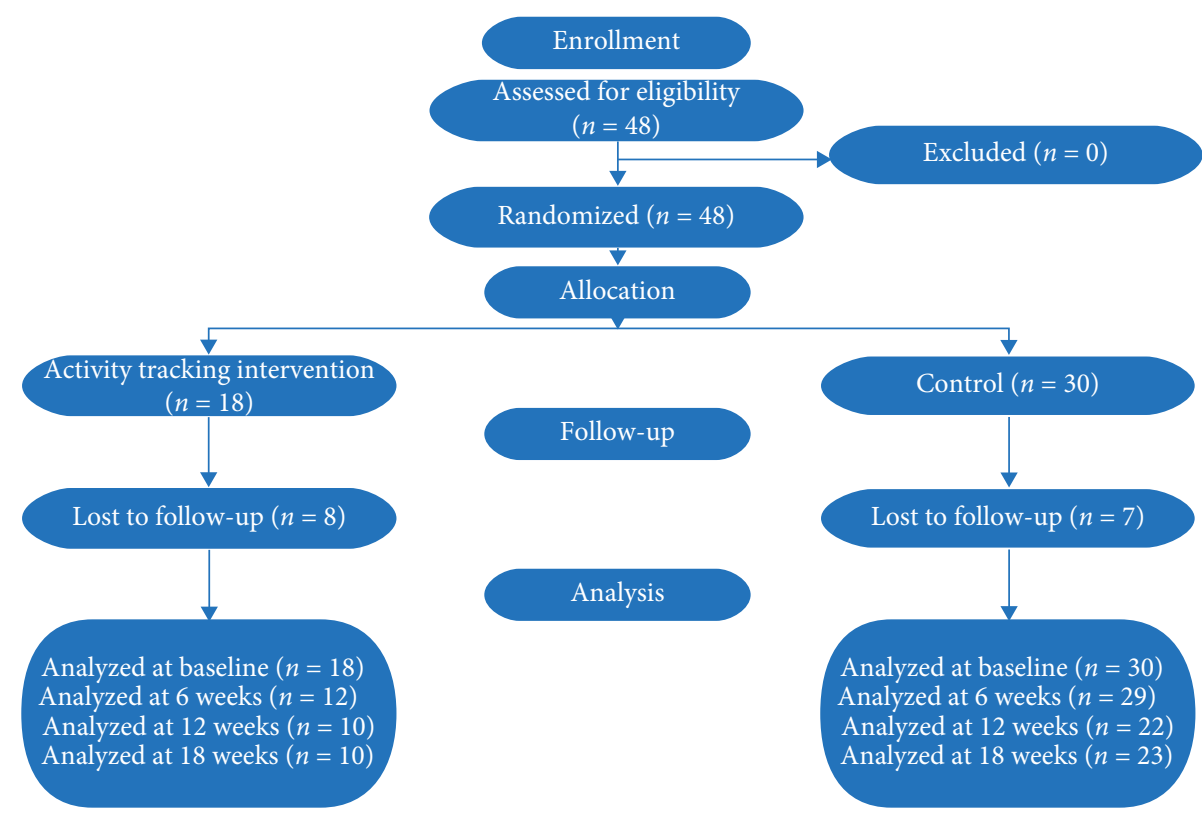

FIgURE 1: Consolidated standards of reporting trials (CONSORT) diagram for pilot randomized trials.

speak English. After written informed parental consent and written informed participant assent, eligible subjects were randomized during a 12-month enrollment period. Randomization was done using $\mathrm{R}$ software and the process accounted for racial distribution. Ethnicities/races were categorized as Hispanic (56\%), White (29\%), and Black (15\%), and block randomization was used for each ethnicity/ race. Within each block, 1 (activity tracking intervention (ATI)) and 2 (control) were randomly selected. As shown in Figure 1, there were 48 participants enrolled in the study with 18 in the ATI group and 30 in the control group. This method resulted in a control: intervention ratio of $1.6: 1$ (30: 18). A 12-month enrollment period was budgeted per protocol. Enrollment may have corrected closer to $1: 1$ if enrollment had continued. Only participants assigned to the ATI group received Fitbit Alta. Receipt of a Fitbit Alta was the only difference between groups.

2.4. Activity Tracking Device. A commercial monitoring device (Fitbit Alta) was selected as the technology for monitoring daily step activity. The Fitbit Alta is a wristband worn tracker that tracks steps, distance, calories burned, minutes in activity, and minutes in sleep. This activity tracker device has been studied and found to be highly reliable for step counting at regular pace and accurate with commonly performed activities. Discrete underestimation at faster speeds and overestimation at slower speeds has also been reported [32].

Participants allocated to the intervention group were given a Fitbit Alta device, were asked to wear it during the day, and were encouraged to walk at least 10,000 steps/day for the study's duration. Previous reviews have reported average steps among children between 10,000 and 13,000 steps/day [33]. The participants could also record and track their dietary intake via the Fitbit mobile app, but they were not required to do so. They were given a study hotline telephone number to call if they encountered technical problems using the device and were issued replacement devices if needed. At each visit, the Fitbit was paired to the patient's Fitbit Application, and the information was downloaded by the research team.

2.5. Measurements. Anthropometric measurements were obtained in each group at baseline and during each subsequent clinic visit. Clinic attendance was also recorded. Fitbit data were downloaded during each visit. Considering that engagement and treatment adherence is one of the principal barriers in successful completion of weight management programs, we chose attrition (a potential surrogate of engagement) as our feasibility measurement. Reported attrition rates in similar weight management clinics vary from 27 to $73 \%[13,14]$. Using this information, we set a study attrition rate $\leq 50 \%$ as the feasibility measure for the study.

2.6. Anthropometry and Demographics. Anthropometric, demographic, and social data collected included sex, age, race/ethnicity, height, weight, BMI, and waist circumference. Height $(\mathrm{cm})$ and weight $(\mathrm{kg})$ were measured using the same scale and the same stadiometer at each visit. Patients were measured in street clothes with no jackets, no shoes, and empty pockets. Obesity was defined as BMI percentile greater than or equal to the 95th percentile. Waist circumference was measured at the uppermost lateral border of the hip crest (ilium).

2.7. Outcomes. The primary outcomes of interest were feasibility (attrition $\leq 50 \%$ ) and absolute change in BMI. In addition, average daily steps were calculated by using data 
downloaded from the activity tracking devices when available. Only days with measurements of 1,000 steps or more were included in the analysis.

2.8. Statistical Analyses. Participants that had a data point at 6,12 , or 18 weeks were included in the statistical analysis, and a 2.5-week margin was permitted. Differences between groups at 6,12 , and 18 weeks were examined using repeated measures analysis. Continuous variables were tested with independent $t$-test, and categorical variables were tested with the chi-square test. Independent $t$-test $p$ value was adjusted by Bonferroni family-wise error adjustment. Fisher's exact test was used when $25 \%$ of cells had less than five expected counts. Analyses were conducted using Stata SE version 15.1.

\section{Results}

3.1. Patient Demographics. Forty-eight participants were enrolled in the study, and baseline demographics and comorbidities were evaluated (Table 1). The average age at baseline evaluation was $14.5( \pm 1.7)$ years, with no difference between treatment and control. Overall, the plurality of participants enrolled was Hispanic (56\%), followed by White (29\%) and Black (15\%). Sexes were equally distributed at baseline with $50 \%$ male and 50\% female. The most common comorbidities were asthma (18.8\%) and OSA (10.4\%), followed by HTN (8.3\%) and DM2 (4.2\%).

3.2. Study Retention. As illustrated in Figure 1, at baseline, there were 48 patients enrolled in the pilot study with 18 in the ATI group and 30 in the control group. Overall attrition at the 6 and 12 week assessment was $39.6 \%$ and $54.2 \%$, respectively. Attrition rate at the study conclusion was $52.1 \%$. The attrition rate was $44.4 \%$ in the ATI group versus $56.6 \%$ in the control group, with a differential attrition of $12.2 \%$. This difference was not statistically significant $(p=0.55)$. The study only demonstrated feasibility (attrition $\leq 50 \%)$ at 6 weeks.

3.3. Anthropometric and Clinical Factors. The average BMI at initial evaluation was $37.5 \mathrm{~kg} / \mathrm{m}^{2}$ (Table 2). There were no significant differences in BMI or weight between the ATI group compared to control at baseline, 6, 12, or 18 weeks (Table 2). Waist circumference was also similar between both groups at any time point.

Absolute changes in BMI and weight are represented in Table 2. Comparison of these variables at each time point showed no significant difference. The ATI and control groups both showed an absolute decrease in BMI at 12 and 18 weeks when compared to baseline. Absolute changes in weight occurred in the ATI group at week 6 and the control group at 18 weeks when compared to baseline.

3.4. Average Steps per Day. As shown in Table 3, the average steps per day during the first week of enrollment were 6,221. At 6 and 12 weeks, the average number of steps decreased by $22.5 \%$ (5,124 steps/day) and by $46.9 \%$ (4,035 steps/day), respectively, as compared to baseline for those who continued participating in the study. At 18 weeks, the average number of steps increased by $1.7 \%$ (6,026 steps/day) from baseline.

\section{Discussion}

This randomized controlled pilot trial was designed to test the feasibility of an ATI in combination with a multidisciplinary adolescent weight management clinic. When considering interventions in a potentially low-compliance population, such as adolescents, feasibility studies performed prior to the development of large clinical trials are important. Delivery of effective weight loss and maintenance strategies in adolescents with obesity is uniquely challenging due to parental dependence, a relatively nonadherent population, and complex sociodemographic factors. These factors contribute to attrition rates as high as $70 \%$ in multidisciplinary weight management clinics.

Clinical trials that evaluate the effectiveness of behavioral technology interventions in adolescent, clinicbased weight management programs are limited. Our study is the first to conduct a randomized pilot trial using activity tracking in a diverse group of adolescents with obesity participating in a multidisciplinary weight management program. Our adolescent population mirrors the adolescent population that suffers from obesity in the United States, with $71 \%$ of those enrolled in the study identifying as Hispanic or Black. Adolescent obesity is nearly twice as common in the non-Hispanic Black (22.0\%) and Hispanic (26\%) populations than the nonHispanic White (14\%) and Asian (11.0\%) populations [4]. The cause of this striking disparity is multifactorial and includes poor access to healthcare, cultural differences, socioeconomic status, and home environment [2, 34, 35]. However, we did not explore the impact of sociodemographic factors on attrition in our study.

The effectiveness of weight management programs is related to how long a patient remains enrolled and engaged, but programs across the board have attrition rates from 27 to $73 \%[13,14]$. In addition, program adherence by minorities and low-income populations presents a particular challenge due to cultural perspectives, community acceptance, childcare concerns, and financial constraints [36, 37]. The attrition rate at the conclusion of our study was $52.1 \%$. The attrition rate was lower in the ATI group (44.4\%) than in the control group (56.6\%); however, this difference did not reach statistical significance. It remains unclear if the decreased attrition rate in the ATI group indicates that the device serves as a reminder to continue study participation. This finding is an avenue for future research in participant retention.

The increase in an individual's ability to track their PA information has not had an identifiable impact on the rising rates of obesity in the United States [1], indicating that technology alone may not be enough to affect behavioral change. Because of this, we hypothesized that a synergistic effect may be seen in concert with a multidisciplinary clinic where a strong focus is placed on behavioral change. 
TABLE 1: Baseline characteristics.

\begin{tabular}{|c|c|c|c|c|}
\hline & Overall $(n=48)$ & ATI $(n=18)$ & Control $(n=30)$ & $p$ value \\
\hline Age, years, mean, SD & $14.48(1.7)$ & $14.5(1.58)$ & $14.47(1.8)$ & 0.94 \\
\hline $\begin{array}{l}\text { Sex, } n(\%) \\
\text { Male } \\
\text { Female }\end{array}$ & $\begin{array}{l}24(50) \\
24(50)\end{array}$ & $\begin{array}{l}9(50) \\
9(50)\end{array}$ & $\begin{array}{l}15(50) \\
15(50)\end{array}$ & 1.00 \\
\hline $\begin{array}{l}\text { Race/ethnicity, } n(\%) \\
\text { NH white } \\
\text { Hispanic } \\
\text { NH black }\end{array}$ & $\begin{array}{c}14(29.17) \\
27(56.25) \\
7(14.58)\end{array}$ & $\begin{array}{c}5(27.78) \\
10(55.56) \\
3(16.67)\end{array}$ & $\begin{array}{c}9(30) \\
17(56.67) \\
4(13.33)\end{array}$ & $1.00 \mathrm{f}$ \\
\hline $\begin{array}{l}\text { BMI, } \mathrm{kg} / \mathrm{m}^{2} \text { mean, SD } \\
\text { Hypertension, } n(\%) \\
\text { Yes } \\
\text { No }\end{array}$ & $\begin{array}{c}2(4.17) \\
46(95.83)\end{array}$ & $\begin{array}{c}36.12(5.9) \\
1(5.56) \\
17(94.44)\end{array}$ & $\begin{array}{c}38.3(10.38) \\
1(3.33) \\
29(96.67)\end{array}$ & $\begin{array}{c}0.36 \\
1.00 f\end{array}$ \\
\hline $\begin{array}{l}\text { OSA, } n(\%) \\
\text { Yes } \\
\text { No } \\
\end{array}$ & $\begin{array}{l}5(10.42) \\
42(87.5)\end{array}$ & $\begin{array}{c}2(11.76) \\
15(88.24)\end{array}$ & $\begin{array}{c}3(10) \\
27(90)\end{array}$ & $1.00 f$ \\
\hline $\begin{array}{l}\text { Asthma, } n(\%) \\
\text { Yes } \\
\text { No } \\
\end{array}$ & $\begin{array}{c}9(18.75) \\
39(81.25) \\
\end{array}$ & $\begin{array}{c}2(11.11) \\
16(88.89)\end{array}$ & $\begin{array}{c}7(23.33) \\
23(76.67) \\
\end{array}$ & $0.45 f$ \\
\hline $\begin{array}{l}\text { GERD, } n(\%) \\
\text { Yes } \\
\text { No } \\
\end{array}$ & $\begin{array}{c}1(2.08) \\
47(97.92) \\
\end{array}$ & $\begin{array}{c}0(0) \\
18(100)\end{array}$ & $\begin{array}{c}1(3.33) \\
29(96.67) \\
\end{array}$ & $1.00 f$ \\
\hline $\begin{array}{l}\text { Diabetes mellitus, } n(\%) \\
\text { Yes } \\
\text { No }\end{array}$ & $\begin{array}{c}4(8.33) \\
44(91.67)\end{array}$ & $\begin{array}{c}2(11.11) \\
16(88.89)\end{array}$ & $\begin{aligned} 2 & (6.67) \\
28 & (93.33)\end{aligned}$ & $0.62 f$ \\
\hline $\begin{array}{l}\text { Drug use, } n(\%) \\
\text { Yes } \\
\text { No } \\
\end{array}$ & $\begin{array}{c}2(4.17) \\
46(95.83) \\
\end{array}$ & $\begin{array}{c}0(0) \\
18(100)\end{array}$ & $\begin{aligned} & 2(6.67) \\
& 28(93.33) \\
&\end{aligned}$ & $0.52 f$ \\
\hline $\begin{array}{l}\text { Alcohol use, } n(\%) \\
\text { Yes } \\
\text { No }\end{array}$ & $\begin{array}{c}5(10.42) \\
43(89.58)\end{array}$ & $\begin{array}{c}3(16.67) \\
15(83.33)\end{array}$ & $\begin{aligned} 2 & (6.67) \\
28 & (93.33)\end{aligned}$ & $0.35 f$ \\
\hline
\end{tabular}

ATI, activity tracking intervention; NH, non-Hispanic; BMI, body mass index; OSA, obstructive sleep apnea; GERD, gastroesophageal reflux disease continuous variables are tested with independent $t$-test. Categorical variables are tested with chi-square test. Fisher's exact test $(f)$ was used when $25 \%$ of cells had less than 5 expected counts.

TABLE 2: Comparison between groups at different time points. Independent $t$-test $p$ value is adjusted by Bonferroni family-wise error adjustment.

\begin{tabular}{|c|c|c|c|c|}
\hline $\begin{array}{l}\text { Baseline } \\
\text { Measure (SD) }\end{array}$ & $\operatorname{ATI}(n=18)$ & Control $(n=30)$ & Total $(n=48)$ & $p$ value \\
\hline Weight, kg & $98.53(21.02)$ & $104.28(32.48)$ & $102.12(28.61)$ & 0.06 \\
\hline Waist circumference, $\mathrm{cm}$ & $110.89(9.94)$ & $114.1(16.42)$ & $112.82(14.03)$ & 0.59 \\
\hline $\mathrm{ALT}, \mathrm{U} / \mathrm{L}$ & $48(27.55)$ & $53.92(58.07)$ & $51.7(48.54)$ & 0.67 \\
\hline $\mathrm{AST}, \mathrm{U} / \mathrm{L}$ & $31.33(12.89)$ & $37.92(37.47)$ & $35.45(30.56)$ & 0.43 \\
\hline ALP, U/L & $145.73(88.75)$ & $161.71(84.09)$ & $155.56(85.11)$ & 0.58 \\
\hline GGT, U/L & $28.07(17.76)$ & $29.5(26.61)$ & $28.97(23.48)$ & 0.86 \\
\hline $\mathrm{CRP}, \mathrm{mg} / \mathrm{L}$ & $0.44(0.24)$ & $0.6(0.45)$ & $0.54(0.39)$ & 0.16 \\
\hline \multicolumn{5}{|l|}{6 weeks } \\
\hline Measure (SD) & ATI $(n=12)$ & Control $(n=17)$ & Total $(n=29)$ & $p$ value \\
\hline BMI, $\mathrm{kg} / \mathrm{m}^{2}$ & $34.84(5.92)$ & $39.89(11.56)$ & $37.8(9.82)$ & 0.41 \\
\hline Change in BMI & $-1.28(-7.4-4.84)$ & $+1.59(-7.13-10.31)$ & $+0.32(-5.36-5.99)$ & \\
\hline Weight, $\mathrm{kg}$ & $96.84(23.81)$ & $107.51(36.16)$ & $103.09(31.6)$ & 1.00 \\
\hline Change in weight & $-1.69(-25.1-21.71)$ & $3.23(-24.59-31.04)$ & $0.97(-17.68-19.61)$ & \\
\hline Waist, cm & $115.43(20.54)$ & $115.4(26.35)$ & $115.41(22.74)$ & 1.00 \\
\hline $\mathrm{ALT}, \mathrm{U} / \mathrm{L}$ & $40.3(14.2)$ & $64.71(68.37)$ & $54.54(52.74)$ & 1.00 \\
\hline AST, U/L & $40(16.92)$ & $41.71(38.58)$ & $41.09(31.3)$ & 1.000 \\
\hline ALP, U/L & $173.63(86.95)$ & $137.86(102.75)$ & $150.86(94.49)$ & 1.00 \\
\hline GGT, U/L & $27.83(9.52)$ & $38.33(43.11)$ & $34.83(34.81)$ & 1.00 \\
\hline $\mathrm{CRP}, \mathrm{mg} / \mathrm{L}$ & $0.62(0.28)$ & $0.34(0.11)$ & $0.44(0.22)$ & 0.26 \\
\hline
\end{tabular}


TABLE 2: Continued.

\begin{tabular}{|c|c|c|c|c|}
\hline \multicolumn{5}{|l|}{12 weeks } \\
\hline BMI, $\mathrm{kg} / \mathrm{m}^{2}$ & $35.43(6.03)$ & $38.03(12.69)$ & $36.85(10.09)$ & 1.00 \\
\hline Change in BMI & $-0.69(-7.17-5.78)$ & $-0.28(-10.09-9.54)$ & $-0.64(-6.85-5.57)$ & \\
\hline Weight, kg & $100.06(24.08)$ & $104.98(39.99)$ & $102.74(33.05)$ & 1.00 \\
\hline Change in weight & $+1.53(-23.24-26.3)$ & $+0.7(-30.6-31.99)$ & $+0.62(-19.79-21.03)$ & \\
\hline Waist, $\mathrm{cm}$ & $106.9(5.37)$ & $110.5(26.45)$ & $108.7(15.72)$ & 1.00 \\
\hline $\mathrm{ALT}, \mathrm{U} / \mathrm{L}$ & $31.75(7.85)$ & $43.33(11.93)$ & $36.71(10.8)$ & 0.53 \\
\hline AST, U/L & $25.25(9.88)$ & $24.67(4.93)$ & $25(7.55)$ & 1.00 \\
\hline ALP, U/L & $92.5(20.04)$ & $97(35.76)$ & $94.43(25.16)$ & 1.00 \\
\hline GGT, U/L & $27.75(11.7)$ & $30.6(10.64)$ & $29.33(10.5)$ & 1.00 \\
\hline $\mathrm{CRP}, \mathrm{mg} / \mathrm{L}$ & $0.5(0.42)$ & $0.85(0.64)$ & $0.68(0.54)$ & 1.00 \\
\hline \multicolumn{5}{|l|}{18 weeks } \\
\hline Measure (SD) & ATI $(n=10)$ & Control $(n=13)$ & Total $(n=23)$ & $p$ value \\
\hline BMI, kg/m² & $35.87(7.01)$ & $35.53(9.39)$ & $35.68(8.26)$ & 1.00 \\
\hline Change in BMI & $-0.25(-6.72-6.23)$ & $-2.77(-12.31-6.76)$ & $-1.81(-7.92-4.31)$ & \\
\hline Weight, kg & $102.7(27.01)$ & $99.15(33.15)$ & $100.69(30.02)$ & 1.00 \\
\hline Change in weight & $+4.17(-20.6-28.94)$ & $-5.13(-35.56-25.29)$ & $-1.43(-21.54-18.67)$ & \\
\hline Waist, cm & $101.3(3.05)$ & $114.2(22.8)$ & $109.36(18.56)$ & 0.83 \\
\hline $\mathrm{ALT}, \mathrm{U} / \mathrm{L}$ & $62(20.66)$ & $97(138.84)$ & $83.88(107.08)$ & 1.00 \\
\hline AST, U/L & $49(6.24)$ & $67.2(94.53)$ & $60.38(72.15)$ & 1.00 \\
\hline ALP, U/L & $141.67(106.02)$ & $160.2(105.22)$ & $153.25(98.13)$ & 1.00 \\
\hline GGT, U/L & $25.67(16.86)$ & $42.2(59.24)$ & $36(46.47)$ & 1.00 \\
\hline $\mathrm{CRP}, \mathrm{mg} / \mathrm{L}$ & $0.4(0.14)$ & $0.2(0.1)$ & $0.28(0.15)$ & 0.46 \\
\hline
\end{tabular}

ATI, activity tracking intervention; BMI, body mass index; GGT, gamma-glutamyl transferase; CRP, C-reactive protein; AST, aspartate aminotransferase; ALT, alanine aminotransferase.

TABLE 3: ATI average daily steps and changes over time.

\begin{tabular}{lcccc}
\hline & Week 1 $(n=16)$ & Week 6 $(n=13)$ & Week 12 $(n=11)$ & Week 18 $(n=7)$ \\
\hline Average steps/day (relative change) & $6,222(3822)$ & $5,124(3969)$ & $4,035(3137)$ & $6,027(3396)$ \\
& Week 1 & $6,615(-2.5 \%, p=0.13)$ & $7,596(-6.9 \%, p=0.02)$ & $5,923(+1.7 \%, p=1.00)$ \\
& & Week 6 & $6,053(-3.3 \%, p=0.20)$ & $5,725(+5.2 \%, p=0.9)$ \\
& & Week 12 & $3,869(+55.7 \%, p=0.10)$ \\
\hline
\end{tabular}

Steps were averaged each day for 1-, 6-, 12-, and 18-week time points for each participant. $p$ values calculated by the paired $t$-test comparing current and previous time point for the participants.

Unfortunately, this type of behavioral technology did not increase daily steps in our study. Although advised to walk 10,000 steps per day, participants only averaged 6,222 steps/ day during the first week, and this did not significantly improve over the study duration. This is consistent with previous studies that did not observe significant increases over time with activity tracking devices [38]. However, current guidelines for the prevention of obesity in childhood and adolescence continue to recommend 60 minutes of physical activity per day [39], and Tudor-Locke et al. [33] reported average steps among children between 10,000 and 13,000 steps/day.

This study had several limitations. Although we set attrition at $\leq 50 \%$ as the feasibility measure for the study, this mark was only met at the 6-week evaluation. Selection bias could have contributed to the study outcomes. The small size, unequal allocation ratio, and attrition in our study might have hindered our ability to detect differences between groups and to assess potential effects of the intervention. In addition, we did not assess participants' readiness to lose weight prior to enrollment in the study or the AWMC. Automated phone calls were placed to parents
24 hours prior to clinic appointments. However, we did not include additional measures to improve compliance. Additional measures might include consultation with an exercise physiologist to analyze the activity tracking device data and modify physical activity plans accordingly. Competitions among participating adolescents using the tracking device could also be considered as a strategy to increase engagement and physical activity.

\section{Conclusion}

Recognizing and changing the societal and cultural factors which influence adolescent obesity is a daunting task. By identifying potential adjunct behavioral interventions, we can strive to provide treatments applicable to all adolescents struggling with obesity. Although we found that the use of a wearable tracking device was not associated with improved attrition or greater weight management in our study, our results could provide critical information for the design of an effective strategy that incorporates enhanced behavioral technology and a multidisciplinary weight management program in the treatment of adolescent obesity. 


\section{Data Availability}

The deidentified clinical data used to support the findings of this study are available from the corresponding author upon request.

\section{Disclosure}

The content is solely the responsibility of the authors and does not necessarily represent the official views of the $\mathrm{Na}$ tional Institutes of Health. The remaining authors have no financial relationships relevant to this article to disclose.

\section{Conflicts of Interest}

The authors have no conflicts of interest to disclose.

\section{Acknowledgments}

Kanika Bowen-Jallow and Erika Fuchs are supported by a research career development award (K12HD052023: Building Interdisciplinary Research Careers in Women's Health Program-BIRCWH; PI: Berenson) from the Eunice Kennedy Shriver National Institute of Child Health and Human Development (NICHD) at the National Institutes of Health.

\section{Supplementary Materials}

Supplement 1: inclusion and exclusion criteria. (Supplementary Materials)

\section{References}

[1] A. C. Skinner, E. M. Perrin, and J. A. Skelton, "Prevalence of obesity and severe obesity in US children, 1999-2014," Obesity, vol. 24, no. 5, pp. 1116-1123, 2016.

[2] S. S. Hawkins, M. W. Gillman, S. L. Rifas-Shiman, K. P. Kleinman, M. Mariotti, and E. M. Taveras, "The Linked CENTURY Study: linking three decades of clinical and public health data to examine disparities in childhood obesity," $B M C$ Pediatrics, vol. 16, p. 32, 2016.

[3] C. M. Hales, M. D. Carroll, C. D. Fryar, and C. L. Ogden, "Prevalence of obesity among adults and youth: United States, 2015-2016," NCHS Data Brief, vol. 288, pp. 1-8, 2017.

[4] C. L. Ogden, M. D. Carroll, B. K. Kit, and K. M. Flegal, "Prevalence of obesity and trends in body mass index among US children and adolescents, 1999-2010," JAMA, vol. 307, no. 5, pp. 483-490, 2012.

[5] O. Nunez Lopez, D. C. Jupiter, F. J. Bohanon, R. S. Radhakrishnan, and K. A. Bowen-Jallow, "Health disparities in adolescent bariatric surgery: nationwide outcomes and utilization," Journal of Adolescent Health, vol. 61, no. 5, pp. 649-656, 2017.

[6] R. A. Miech, S. K. Kumanyika, N. Stettler, B. G. Link, J. C. Phelan, and V. W. Chang, "Trends in the association of poverty with overweight among US adolescents, 1971-2004," JAMA, vol. 295, no. 20, pp. 2385-2393, 2006.

[7] C. B. Cummins, O. Nunez Lopez, B. D. Hughes et al., "Adolescent bariatric surgery: effects of socioeconomic, demographic, and hospital characteristics on cost, length of stay, and type of procedure performed," Obesity Surgery, vol. 29, no. 3, pp. 757-764, 2019.

[8] T. Lobstein, R. Jackson-Leach, M. L. Moodie et al., "Child and adolescent obesity: part of a bigger picture," The Lancet, vol. 385, no. 9986, pp. 2510-2520, 2015.

[9] L. Y. Wang, M. Denniston, S. Lee, D. Galuska, and R. Lowry, "Long-term health and economic impact of preventing and reducing overweight and obesity in adolescence," Journal of Adolescent Health, vol. 46, no. 5, pp. 467-473, 2010.

[10] Y. C. Wang, J. Pamplin, M. W. Long, Z. J. Ward, S. L. Gortmaker, and T. Andreyeva, "Severe obesity in adults cost state medicaid programs nearly $\$ 8$ billion in 2013," Health Affairs, vol. 34, no. 11, pp. 1923-1931, 2015.

[11] M. Savoye, M. Shaw, J. Dziura et al., "Effects of a weight management program on body composition and metabolic parameters in overweight children," JAMA, vol. 297, no. 24, pp. 2697-2704, 2007.

[12] A. J. Cochrane, B. Dick, N. A. King, A. P. Hills, and D. J. Kavanagh, "Developing dimensions for a multicomponent multidisciplinary approach to obesity management: a qualitative study," BMC Public Health, vol. 17, no. 1, p. 814, 2017.

[13] S. E. Hampl, K. B. Borner, K. M. Dean et al., "Patient attendance and outcomes in a structured weight management program," The Journal of Pediatrics, vol. 176, pp. 30-35, 2016.

[14] J. A. Skelton and B. M. Beech, "Attrition in paediatric weight management: a review of the literature and new directions," Obesity Reviews, vol. 12, no. 5, pp. e273-e281, 2011.

[15] E. T. Rhodes, R. E. Boles, K. Chin et al., "Expectations for treatment in pediatric weight management and relationship to attrition," Childhood Obesity, vol. 13, no. 2, pp. 120-127, 2017.

[16] B. J. Sallinen Gaffka, M. Frank, S. Hampl, M. Santos, and E. T. Rhodes, "Parents and pediatric weight management attrition: experiences and recommendations," Childhood Obesity, vol. 9, no. 5, pp. 409-417, 2013.

[17] T. T. Phan, N. Barnini, S. Xie et al., "Feasibility of using a commercial fitness tracker as an adjunct to family-based weight management treatment: pilot randomized trial," JMIR Mhealth Uhealth, vol. 6, no. 11, Article ID e10523, 2018.

[18] A. N. Sullivan and M. E. Lachman, "Behavior change with fitness technology in sedentary adults: a review of the evidence for increasing physical activity," Frontiers in Public Health, vol. 4, p. 289, 2016.

[19] A. Henriksen, M. Haugen Mikalsen, A. Z. Woldaregay et al., "Using fitness trackers and smartwatches to measure physical activity in research: analysis of consumer wrist-worn wearables," Journal of Medical Internet Research, vol. 20, no. 3, p. e110, 2018.

[20] J. Xie, D. Wen, L. Liang, Y. Jia, L. Gao, and J. Lei, "Evaluating the validity of current mainstream wearable devices in fitness tracking under various physical activities: comparative study," JMIR Mhealth and Uhealth, vol. 6, no. 4, p. e94, 2018.

[21] H.-S. An, G. C. Jones, S.-K. Kang, G. J. Welk, and J.-M. Lee, "How valid are wearable physical activity trackers for measuring steps?” European Journal of Sport Science, vol. 17, no. 3, pp. 360-368, 2017.

[22] C. Maher, J. Ryan, C. Ambrosi, and S. Edney, "Users' experiences of wearable activity trackers: a cross-sectional study," BMC Public Health, vol. 17, no. 1, p. 880, 2017.

[23] S. P. Wright, T. S. Hall Brown, S. R. Collier, and K. Sandberg, "How consumer physical activity monitors could transform human physiology research," American Journal of PhysiologyRegulatory, Integrative and Comparative Physiology, vol. 312, no. 3, pp. R358-R367, 2017. 
[24] A. Pourzanjani, T. Quisel, and L. Foschini, "Adherent use of digital health trackers is associated with weight loss," PLoS One, vol. 11, no. 4, Article ID e0152504, 2016.

[25] J. A. Naslund, K. A. Aschbrenner, E. A. Scherer, G. J. McHugo, L. A. Marsch, and S. J. Bartels, "Wearable devices and mobile technologies for supporting behavioral weight loss among people with serious mental illness," Psychiatry Research, vol. 244, pp. 139-144, 2016.

[26] M. A. Adams, J. C. Hurley, M. Todd et al., "Adaptive goal setting and financial incentives: a $2 \times 2$ factorial randomized controlled trial to increase adults' physical activity," $B M C$ Public Health, vol. 17, no. 1, p. 286, 2017.

[27] L. Gualtieri, S. Rosenbluth, and J. Phillips, "Can a free wearable activity tracker change behavior? The impact of trackers on adults in a physician-led wellness group," JMIR Research Protocols, vol. 5, no. 4, p. e237, 2016.

[28] J. Bianchi-Hayes, E. Schoenfeld, R. Cataldo, W. Hou, C. Messina, and S. Pati, "Combining activity trackers with motivational interviewing and mutual support to increase physical activity in parent-adolescent dyads: longitudinal observational feasibility study," JMIR Pediatrics and Parenting, vol. 1, no. 1, p. e3, 2018.

[29] N. D. Ridgers, M. A. McNarry, and K. A. Mackintosh, "Feasibility and effectiveness of using wearable activity trackers in youth: a systematic review," JMIR mHealth and uHealth, vol. 4, no. 4, p. e129, 2016.

[30] E. A. Finkelstein, B. A. Haaland, M. Bilger et al., "Effectiveness of activity trackers with and without incentives to increase physical activity (TRIPPA): a randomised controlled trial," The Lancet Diabetes \& Endocrinology, vol. 4, no. 12, pp. 983-995, 2016.

[31] T. Reinehr, "Lifestyle intervention in childhood obesity: changes and challenges," Nature Reviews Endocrinology, vol. 9, no. 10, pp. 607-614, 2013.

[32] K. R. Evenson, M. M. Goto, and R. D. Furberg, "Systematic review of the validity and reliability of consumer-wearable activity trackers," International Journal of Behavioral Nutrition and Physical Activity, vol. 12, p. 159, 2015.

[33] C. Tudor-Locke, C. L. Craig, M. W. Beets et al., "How many steps/day are enough? For children and adolescents," International Journal of Behavioral Nutrition and Physical Activity, vol. 8, no. 1, p. 78, 2011.

[34] M. G. Orr, S. Galea, M. Riddle, and G. A. Kaplan, "Reducing racial disparities in obesity: simulating the effects of improved education and social network influence on diet behavior," Annals of Epidemiology, vol. 24, no. 8, pp. 563-569, 2014.

[35] C. F. Haughton, M. L. Wang, and S. C. Lemon, "Racial/ethnic disparities in meeting 5-2-1-0 recommendations among children and adolescents in the United States," The Journal of Pediatrics, vol. 175, pp. 188-194, 2016.

[36] Z. Cui, E. M. Seburg, N. E. Sherwood, M. S. Faith, and D. S. Ward, "Recruitment and retention in obesity prevention and treatment trials targeting minority or low-income children: a review of the clinical trials registration database," Trials, vol. 16, p. 564, 2015.

[37] M. Zeller, S. Kirk, R. Claytor et al., "Predictors of attrition from a pediatric weight management program," The Journal of Pediatrics, vol. 144, no. 4, pp. 466-470, 2004.

[38] J. B. Wang, L. A. Cadmus-Bertram, L. Natarajan et al., "Wearable sensor/device (Fitbit one) and SMS text-messaging prompts to increase physical activity in overweight and obese adults: a randomized controlled trial," Telemedicine and E-Health, vol. 21, no. 10, pp. 782-792, 2015.
[39] US Department of Health and Human Services, "Active children and adolescents. 2008 physical activity guidelines for Americans. (ODPHP publication U0036)," pp. 15-21, http:// health.gov/paguidelines/guidelines. 